\title{
Long-term Functional Outcomes and Relapse of Anti-NMDA Receptor Encephalitis
}

\section{A Cohort Study in Western China}

Xue Gong, MD, Chu Chen, MD, Xu Liu, MD, PhD, Jingfang Lin, MD, Aiqing Li, MD, Kundian Guo, MD, Dong Zhou, MD, PhD,* and Zhen Hong, MD, PhD*

Neurol Neuroimmunol Neuroinflamm 2021;8:e958. doi:10.1212/NXI.0000000000000958

\section{Abstract}

\section{Objective}

To study the factors associated with relapse and functional outcomes in patients with antiNMDA receptor encephalitis in Western China.

\section{Methods}

The Outcome of the anti-NMDA receptor Encephalitis Study in Western China was initiated in October 2011 to collect prospective observational data from consecutively enrolled patients with anti-NMDA receptor encephalitis.

\section{Results}

We consecutively enrolled 244 patients (median age: 26 years, range: 9-78 years; females: 128 [52.45\%]) between October 2011 and September 2019. Fatality occurred in 17 (6.96\%) patients, and tumors were found in $38(15.57 \%)$ patients. The median follow-up duration was 40 (6-96) months. Of these patients, $84.8 \%$ showed clinical improvements within 4 weeks after immunotherapy, with a median modified Rankin Scale of 2 (interquartile range [IQR]: 2-3), and $80.7 \%$ (median: 1, IQR: $0-2$ ) and $85.7 \%$ (median: 0 , IQR: $0-1$ ) had substantial recovery (i.e., mild or no residual symptoms) at 12 and 24 months, respectively. The overall prognosis was still improving at 42 months after onset. Disturbance of consciousness during the first month was the only independent predictor (OR: 2.91, 95\% CI: $1.27-6.65 ; p=0.01$ ) of a poor functional neurologic outcome. Overall, $15.9 \%$ of the patients had one or multiple relapses, with 82.0\% experiencing the first relapse within 24 months and $76.9 \%$ experiencing relapses that were less severe than the initial episodes. Relapse-related risk factors included the female sex and delayed treatment $(p<0.05)$.

\section{Conclusions}

Most patients achieved favorable long-term functional outcomes. Some patients experienced one or multiple relapses, especially female patients. Timely immunotherapy at onset may reduce the risk of relapse.

\author{
Correspondence \\ Dr. Hong \\ hongzhengoog@aliyun.com \\ or Dr. Zhou \\ zhoudong66@yahoo.de
}




\section{Glossary}

$\mathbf{H R}=$ hazard ratio; $\mathbf{I C U}=$ intensive care unit; $\mathbf{I g G}=$ immunoglobulin $\mathrm{G} ; \mathbf{I Q R}=$ interquartile range; IVIg = IV immunoglobulin; IVMP = IV methylprednisolone; $\mathbf{M M F}=$ mycophenolate mofetil; $\mathbf{m R S}=$ modified Rankin Scale; NMDAR = NMDA receptor; ONE-WC = Outcome of anti-NMDAR Encephalitis Study in Western China; RTX = rituximab; SE = status epilepticus.

Anti-NMDA receptor (NMDAR) encephalitis is a recently identified autoimmune disorder with characteristic clinical features, ${ }^{1}$ which was first described in 2007 by Dalmau and Bataller. $^{2}$ The target antigens in this disorder are neuronal cell-surface proteins, and all patients have immunoglobulin G ( $\mathrm{IgG})$ antibodies against the GluN1 subunit of the NMDAR ${ }^{3}$ Most patients experience a viral-like prodrome (e.g., fever and headache) as a prodromal symptom, followed by the development of severe psychiatric symptoms, memory loss, seizures, decreased consciousness, and dyskinesias. ${ }^{4}$ Despite the expanding knowledge base, the factors underlying disease prognosis and relapse are poorly understood.

Previous reports describing the clinical characteristics, radiologic features, tumor associations, treatment strategies, and relapse rates have varied across populations. ${ }^{3,5,6}$ Previous reports in the United States and Europe ${ }^{7-9}$ showed that more than $80 \%$ of patients were female, and $20 \%-59 \%$ of patients had tumors. Our previous studies revealed a relatively higher proportion of males (45.0\%) and reduced tumor rates (15.0\%); furthermore, only $15 \%$ of Chinese patients were admitted to the intensive care unit (ICU), compared with 50\%-77\% in the other cohorts. ${ }^{10,11}$ The relapse rate in our study was $15.95 \%$, whereas that in other studies varied between $7.8 \%$ and $25 \%,{ }^{12,13}$ even reaching $36.4 \% .{ }^{14}$ These phenomena may suggest that anti-NMDAR encephalitis is heterogeneous among people of different races, which could be due to the differences in genetic backgrounds or due to the etiology of the disease. To date, large cohort studies on the factors associated with relapse and functional outcomes in patients with anti-NMDAR encephalitis in Western China are lacking.

To obtain estimates of the functional outcomes associated with anti-NMDAR encephalitis in Western China, a study named the Outcome of anti-NMDAR Encephalitis Study in Western China (ONE-WC study) was initiated in October 2011 to collect prospective observational data from consecutively enrolled patients with anti-NMDAR encephalitis. As part of the ONE-WC study, this study updates the clinical profiles of patients with antiNMDAR encephalitis in a large cohort size with a 9-year followup. In addition, we particularly focused on patient functional outcomes, relapse phenomena, and factors that may predict and affect the risk of relapse.

\section{Methods}

\section{Study Design and Participants}

The ONE-WC study was registered with the World Health Organization international clinical trial registry platform (registration number: ChiCTR1800019762) and was described in more detail in our previous publications. ${ }^{11,15-17}$ Briefly, the ONE-WC study consecutively enrolled patients with anti-NMDAR encephalitis from the Department of Neurology at West China Hospital beginning in October 2011 and collected prospective observational data (e.g., demographic information, clinical characteristics, treatment strategy, and functional outcomes). Participants in this study were recruited between October 2011 and September 2019. We included patients who satisfied the criteria for definite anti-NMDAR encephalitis according to definitions of autoimmune encephalitis from a recent consensus statement. ${ }^{18}$ The inclusion criteria were as follows ${ }^{18}$ : (1) acute onset of 1 or more of the following 8 major groups of manifestations: psychosis, memory deficit, speech disturbance, seizures, movement disorder, disturbance of consciousness, autonomic dysfunction, and central hypoventilation; (2) CSF tests positive for NMDAR antibodies (cell-based assay); and (3) reasonable exclusion of other disorders.

The exclusion criteria were as follows: (1) patients with HIV infection, brain abscess, prion diseases, cerebral malaria, brain tumor, or a diagnosis of a noninfectious CNS disease, such as acute demyelinating encephalomyelitis; (2) patients with CNS viral, bacterial, fungal, parasitic, or Mycobacterium tuberculosis infection; (3) patients with encephalopathy secondary to sepsis or systemic inflammatory response syndrome; (4) patients diagnosed with epilepsy, cerebral trauma, and/or other nervous system diseases before the onset of encephalitis; (5) patients with positive serum and/or CSF laboratory tests for another autoimmune encephalitis: a-amino-3-hydroxy-5-methyl-4isoxazol-propionic acid receptor antibody encephalitis, contactin-associated protein 2 antibody encephalitis, leucinerich glioma-inactivated protein 1 antibody encephalitis, gamma-aminobutyric acid receptors $\mathrm{B} 1 / \mathrm{B} 2$ receptor antibody encephalitis, voltage-gated potassium channel complex antibody encephalitis, and glutamate decarboxylase antibody encephalitis; and (6) patients lacking key clinical data.

\section{Data Collection}

The statuses of patients in the acute stage were obtained from hospital medical records, and face-to-face interviews were conducted by experienced neurologists. The standardized data collection included (1) epidemiologic data such as sex, age at disease onset, education, location, and ethnicity; (2) clinical data such as typical symptoms (such as psychosis, memory deficits, speech disturbances, seizures, movement disorders, disturbance of consciousness, autonomic dysfunction, and central hypoventilation), cooccurrence of symptoms such as fever, headache, 
dizziness, ICU admission (including severe anti-NMDAR encephalitis with ovarian teratoma requiring surgical operation, status epilepticus [SE], mechanical ventilation requirement, and autonomic instability), and other clinical phenotypes at onset, number of relapses, clinical phenotype at relapse and at last follow-up, and time to first relapse; (3) biological data such as CSF cell count (pleocytosis $>5$ cells $/ \mathrm{mm}^{3}$ ), open pressure, glucose, chloride, and immunoglobulin G index; (4) MRI and EEG results; (5) immunotherapies including first-line treatments (corticosteroids, IV immunoglobulin [IVIg], and plasmapheresis alone or in combination), second-line treatments (rituximab [RTX] and cyclophosphamide [CTX] alone or in combination), ${ }^{5,18}$ and long-term immunotherapies (immunosuppressants [mycophenolate mofetil (MMF), tacrolimus, and CTX] or low-dose corticosteroids for at least 3 months). ${ }^{7}$

\section{Evaluation Prognosis and Operational Definitions}

Follow-up information was evaluated every 3 months after disease onset by a clinician and objectively assessed by the treating neurologist. Clinical relapse was defined as new onset or worsening of symptoms occurring after an initial improvement or stabilization of at least 2 months. ${ }^{18}$ If patients reported clinical worsening or relapse, an extra visit was scheduled, and they were assessed by a relapse questionnaire designed for this study. The questionnaire included the frequency of relapses, number of events, time to relapse, clinical data at relapse, and treatment at relapse. Thorough clinical and laboratory examinations were conducted to rule out other etiologies and to validate the diagnosis of relapse.

Clinical functional outcomes were assessed through the modified Rankin Scale (mRS) ${ }^{19}$ which was used to classify patients who were able to walk without aid $(\mathrm{mRS} \leq 2)$ or needed assistance while walking $(\mathrm{mRS} \geq 3)(0=$ asymptomatic; $1=$ nondisabling symptoms that do not interfere with lifestyle; $2=$ minor disabling symptoms that lead to some restriction of lifestyle but do not prevent totally independent existence; 3 = symptoms significantly interfering with lifestyle or preventing totally independent existence; 4 = moderately severe disabling symptoms that clearly prevent independent existence with total support needed for basic daily activities; $5=$ severe disabling symptoms, totally dependent and requiring constant attention day and night; and $6=$ death due to neurologic symptoms). A poor response to immunotherapy was defined as no improvement in the mRS score or an $\mathrm{mRS}$ score $\geq 4$ for 4 weeks; clinical improvement was defined as a decrease in the $\mathrm{mRS}$ score $\geq 1$ point from that at the previous visit. ${ }^{7,14}$ Long-term favorable and poor functional outcomes were defined as an mRS score of $\leq 2$ and an mRS score of $>2$ at 12 months of follow-up, respectively. ${ }^{7,13}$ Early treatment was defined as the initiation of immunotherapy within 30 days of onset. ${ }^{20} \mathrm{We}$ defined the use of 3 or more different immunotherapies as the combination of at least 3 of the abovementioned agents. ${ }^{21}$

\section{Statistical Analysis}

Data were entered in an Excel spreadsheet and analyzed with SPSS IBM 20.0, and figures were generated with GraphPad
Prism 7 and Origin 2019. Quantitative data with normal distributions are presented as mean $\pm \mathrm{SD}$, otherwise as the median with the range. Symptoms and demographic data were analyzed using the $\chi^{2}$ test, $\chi^{2}$ analysis with continuity correction or Fisher's exact test for categorical variables where applicable, and the Mann-Whitney $U$ test for continuous variables. Factors affecting functional outcomes were assessed using univariate logistic regression. Predictor variables with $p$ values below 0.10 as determined by univariate analysis were included in the multivariate logistic regression analysis, which was used to evaluate independent predictors of long-term functional outcomes for patients with NMDAR encephalitis after adjusting for potential confounding factors.

According to the main objective of the study, demographic, clinical, and treatment data at disease onset were considered predictors of time to relapse. In the analysis of survival from the first event, the follow-up times were as follows: for relapsing patients, the time to first relapse; for relapse-free patients, the time to last follow-up. The follow-up times for the relapse analysis were calculated with the Kaplan-Meier method. ${ }^{22}$ The factors predictive of survival from the first relapse were analyzed by univariate Cox regression. Multivariate analysis was not performed in view of the low number of patients with relapses. ${ }^{23}$ Subgroup analyses of predictive factors were also performed in cases that relapsed early (within 3-6 months) and later. $p<0.05$ was considered significant.

\section{Standard Protocol Approvals, Registrations, and Patient Consent}

Approval for this study was provided by the Research Ethics Committee of the Medical School of Sichuan University. Informed consent was obtained from each patient. All the data analyzed in the study were strictly anonymous.

\section{Data Availability}

The authors confirm that the data supporting the findings of this study are available within the article and from the corresponding author upon reasonable request.

\section{Results}

\section{Demographic and Clinical Characteristics}

A total of 244 patients were enrolled. Table 1 describes the patient demographic and clinical characteristics in detail. The proportions of patients with cumulative clinical symptoms during the first month of disease stratified by different ages at onset are shown in figure 1A. The figure shows increasing trends for the proportions of patients with central ventilation, autonomic symptoms, and cognitive disorders as patients aged. By contrast, the proportions of speech disturbance and seizures decreased with age.

Figure 1B shows the distributions of the presence or absence of tumors in patients by age and sex. Tumors were found in 38 
Table 1 Comparisons of the Clinical Data From Patients With Anti-NMDA Receptor Encephalitis in Different Age Groups No. of patients (\%)

All $(n=244) \quad$ Age $<18(n=43) \quad$ Age $\geq 18(n=201) \quad p$ Value

\section{Demographic data}

\begin{tabular}{|c|c|c|c|c|}
\hline Age, y, median (IQR) & $26(9-78)$ & $16(9-18)$ & $29(19-78)$ & \\
\hline Sex (female) & $128(54.50)$ & $27(49.09)$ & $101(53.43)$ & $0.27^{a}$ \\
\hline \multicolumn{5}{|l|}{ Symptoms ${ }^{e}$} \\
\hline Fever & 95 (38.9) & 19 (43.2) & $76(38.0)$ & $0.43^{a}$ \\
\hline Headache & 89 (36.5) & $5(11.6)$ & $84(41.8)$ & $0.001^{\circ}$ \\
\hline Ataxia & $6(2.5)$ & $1(2.3)$ & $5(2.5)$ & $1.00^{\mathrm{b}}$ \\
\hline Limb weakness & $13(5.3)$ & $1(2.3)$ & $12(6.0)$ & $0.47^{\mathrm{b}}$ \\
\hline Limb numbness & $3(1.2)$ & $1(2.3)$ & $2(1.0)$ & $0.47^{\mathrm{b}}$ \\
\hline Cognitive disorder & $161(66.0)$ & $25(58.1)$ & $136(69.7)$ & $0.14^{\mathrm{a}}$ \\
\hline Dizziness & $17(7.0)$ & $0(0)$ & $17(8.5)$ & $0.04^{c}$ \\
\hline Disturbance of consciousness & $100(41.0)$ & $22(51.2)$ & $78(38.8)$ & $0.13^{a}$ \\
\hline Speech disturbance & $60(24.6)$ & $11(25.6)$ & $49(24.4)$ & $0.86^{a}$ \\
\hline Dyskinesias and movement disorders & $98(40.2)$ & 18 (41.9) & $80(40.0)$ & $0.82^{\mathrm{a}}$ \\
\hline Sleep disorders & $92(37.7)$ & 18 (41.9) & $74(36.8)$ & $0.53^{a}$ \\
\hline Seizures & $186(76.2)$ & $31(72.1)$ & 155 (77.7) & $0.48^{a}$ \\
\hline Focal onset & $91(37.3)$ & $19(47.5)$ & $72(35.8)$ & $0.16^{a}$ \\
\hline Generalized onset & $155(63.5)$ & $27(64.3)$ & $128(63.7)$ & $0.94^{a}$ \\
\hline Epileptic state & 73 (29.9) & $20(47.6)$ & $53(26.4)$ & $0.006^{\circ}$ \\
\hline Psychosis & $222(91.0)$ & $38(88.4)$ & $184(91.5)$ & $0.51^{a}$ \\
\hline Mania & $106(43.4)$ & $20(46.5)$ & $86(42.8)$ & $0.65^{a}$ \\
\hline Depression & $27(11.1)$ & $2(4.7)$ & 25 (12.4) & $0.18^{b}$ \\
\hline Hallucinations & $203(83.2)$ & $36(83.7)$ & $167(83.1)$ & $0.91^{a}$ \\
\hline Autonomic dysfunction, $\geq 1$ of the following & $118(48.4)$ & $18(41.9)$ & $100(49.8)$ & $0.34^{a}$ \\
\hline Hyperhidrosis & $114(46.7)$ & $22(51.2)$ & $92(45.8)$ & $0.52^{\mathrm{a}}$ \\
\hline Tachycardia & $61(25.0)$ & $5(11.6)$ & $56(27.9)$ & $0.02^{a}$ \\
\hline Urinary retention or hesitation & $56(23.0)$ & $7(16.3)$ & $49(24.4)$ & $0.25^{\mathrm{a}}$ \\
\hline Hyponatremia & $38(15.6)$ & $5(11.6)$ & $33(16.4)$ & $0.43^{a}$ \\
\hline Central hypoventilation & $41(16.8)$ & $4(9.3)$ & $37(18.4)$ & $0.14^{b}$ \\
\hline Admission to the intensive care unit & $35(14.3)$ & $2(4.7)$ & $33(16.4)$ & $0.04^{b}$ \\
\hline Complication & $176(72.1)$ & $30(68.2)$ & $146(73.0)$ & $0.31^{\mathrm{a}}$ \\
\hline Tumor & $38(15.6)$ & $7(16.3)$ & $31(15.4)$ & $0.88^{a}$ \\
\hline Teratoma & $27(11.1)$ & $5(11.6)$ & $22(10.9)$ & $0.89^{a}$ \\
\hline \multicolumn{5}{|l|}{ Auxiliary examinations } \\
\hline Abnormal MRI & $104(42.6)$ & $19(44.2)$ & $85(42.2)$ & $0.81^{a}$ \\
\hline Abnormal EEG & $188(77.0)$ & $32(74.4)$ & $156(77.6)$ & $0.65^{a}$ \\
\hline Abnormal CSF & $127(52.0)$ & $20(45.5)$ & $107(53.5)$ & $0.40^{a}$ \\
\hline
\end{tabular}


Table 1 Comparisons of the Clinical Data From Patients With Anti-NMDA Receptor Encephalitis in Different Age Groups (continued)

\begin{tabular}{|c|c|c|c|c|}
\hline & \multicolumn{3}{|c|}{ No. of patients (\%) } & \multirow[b]{2}{*}{$p$ Value } \\
\hline & All $(n=244)$ & Age $<18(n=43)$ & Age $\geq 18(n=201)$ & \\
\hline \multicolumn{4}{|l|}{ Treatment } & $0.06^{\mathrm{c}}$ \\
\hline No treatment & $10(4.1)$ & $0(0.0)$ & $10(5.0)$ & \\
\hline IVIg alone & $80(32.8)$ & $17(39.5)$ & $63(31.3)$ & \\
\hline IVMP alone & $29(12.3)$ & $1(2.3)$ & $28(13.9)$ & \\
\hline IVIg combined with IVMP & $125(50.8)$ & $25(56.8)$ & $100(49.5)$ & \\
\hline$\geq 3$ different immunotherapies & $22(9.0)$ & $3(6.8)$ & $19(9.5)$ & $0.77^{b}$ \\
\hline mRS on admission, median (IQR) & $4(4-5)$ & $4(4-5)$ & $4(4-5)$ & $0.95^{\mathrm{d}}$ \\
\hline Length of hospital stay, d, mean \pm SD & $28.5 \pm 30.1$ & $29.0 \pm 32.2$ & $26.0 \pm 17.6$ & $0.61^{d}$ \\
\hline Poor response to treatment ${ }^{f}$ & $37(15.2)$ & $4(9.3)$ & $33(16.4)$ & $0.23^{\mathrm{b}}$ \\
\hline \multicolumn{5}{|c|}{$\begin{array}{l}\text { Abbreviations: IQR = interquartile range; IVIg = IV immunoglobulin; IVMP = IV methylprednisolone. } \\
\text { Bold entries indicate } p<0.05 \text {. } \\
\text { a Pearson's } \chi^{2} \text { test. } \\
\text { b Chi-squared test with continuity correction. } \\
\text { ' Fisher's exact test. } \\
\text { d Mann-Whitney } U \text { test. } \\
\text { e The cumulative symptoms during the first month of the disease. } \\
{ }^{f} \text { A poor response to immunotherapy was defined as no improvement in the mRS score or an mRS }\end{array}$} \\
\hline
\end{tabular}

(15.6\%) patients and predominated in females $(p<0.05) ; 28$ females had ovarian teratomas and 1 male had mediastinal teratoma. The remaining 9 tumors $(23.1 \%)$ were as follows: 3 lung cancers ( 2 with small cell lung cancer and 1 with lung adenocarcinoma); 2 thyroid tumors; 1 thymoma; 1 brain glioma; 1 adrenal carcinoma, and 1 bladder cancer. All the patients with the remaining 9 tumors were older than 40 years.

Comparisons of the differences between male and female patients are summarized in table e-1 (links.lww.com/NXI/ A412). There was no sex ratio difference in our cohort (female vs male: 128 [52.4\%] vs 116 [47.5\%]). The average age at onset in male patients was younger than that in female patients ( 24.5 vs 30.8 , respectively, $p=0.04)$. Abnormalities in MRI, CSF examinations, and the frequency of seizures were found to be more prevalent in male patients $(p<0.05)$. However, the frequencies of other symptoms, including treatment, immunotherapy response, baseline mRS, and hospitalization time, were not significantly different between males and females.

\section{Neurologic Functional Outcomes and Factors Associated With a Poor Long-term Functional Outcome}

The median follow-up duration was 40 months (6-96 months), and the median mRS scores at baseline and at 4 weeks after the initial immunotherapy were 4 and 2, respectively. To investigate the long-term prognosis of patients with this disease, the mRS scores were evaluated over time and stratified by whether the patients had recurrence, and these results are summarized in figure 1, C-E. Overall, $80.7 \%$ and $85.7 \%$ of patients had attained favorable neurologic functional outcomes (mRS of $0-2$ ) at 12 and 24 months of follow-up, respectively. The overall prognosis was still improving at 42 months after onset. The median mRS score at the 24-month follow-up was 0 , which was significantly lower than the score of 4 (range: $2-5$ ) at onset (Wilcoxon test: $Z=$ $-12.839, p<0.0001)$.

In total, 17 patients died, most of whom died because of disease progression and complications. Seven patients died in the ICU within 6 months of follow-up after disease onset (3 died of sepsis, 2 died of acute respiratory distress, and 2 died of refractory SE). Four patients died after the withdrawal of medical support, and 3 patients committed suicide. Three patients died of other causes ( 1 due to acute myocardial infarction, 1 due to accidental death, and 1 due to pneumonia). All patients with tumors underwent removal surgery, and 1 patient with lung cancer died. Sequela and deficits included speech disturbances $(n=22)$, movement disorders $(n=6)$, psychiatric symptoms $(n=7)$, memory deficits $(n=40)$, and seizures $(n=6)$.

Table 2 summarizes the comparisons between the patients with favorable and poor clinical functional outcomes among 225 patients with anti-NMDAR encephalitis at the 12-month follow-up. The patients with worse neurologic functional outcomes (mRS 3-6) had a higher rate of pleocytosis $(Z=$ 3.63, $p=0.05)$ and higher frequencies of central 

Disease Onset) of 244 Patients With Anti-NMDA Receptor Encephalitis

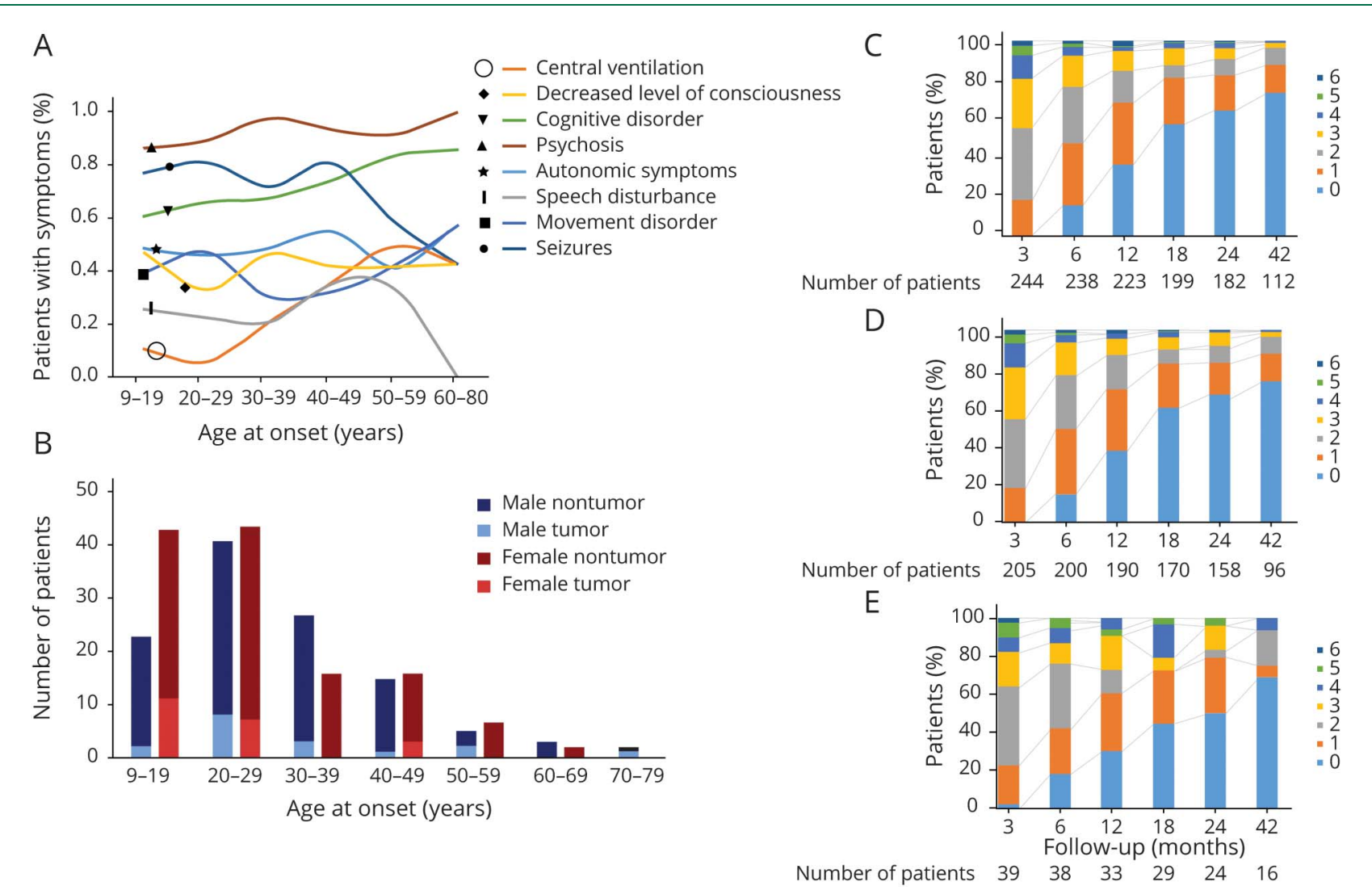

(A) The proportions of patients with cumulative clinical symptoms stratified by different ages at onset. (B) The distributions of patients by age, sex, and the presence or absence of tumors. The modified Rankin Scale scores of the patients with anti-NMDA receptor encephalitis at different follow-up points are shown in (C) for all patients and stratified by patients with (D) a monophasic course and (E) a relapsing course.

hypoventilation $(Z=3.89, p=0.04)$, disturbance of consciousness $(Z=8.49, p=0.04)$, and SE $(Z=3.35, p=0.05)$ during the first month of the disease than the patients with favorable neurologic functional outcomes (mRS 0-2). Finally, disturbance of consciousness was the only independent predictor after performing multivariate logistic regression analysis (OR: 2.91, 95\% CI: $1.27-6.65 ; p=0.01$ ). Multivariate analyses of independent predictors of poor functional outcomes are shown in table e-2 (links.lww.com/NXI/A412). Although there were no significant differences in the rates of ICU admission and the presence of tumors between the 2 groups, these parameters were higher in patients with poor functional outcomes than in those with favorable functional outcomes.

\section{Relapse}

In total, 39 patients $(39 / 244,15.9 \%)$ relapsed. The median duration from onset to the first relapse was 11 (range: 2-38) months. During the first 24 months, 32 (82.0\%) patients experienced an initial relapse. However, after 3 years of onset, 1 female patient in our cohort still relapsed. The median age at relapse was 26 (range: $12-71$ ) years. Of the relapsed patients, 27 (65.8\%) were female, and 2 had ovarian teratomas and tumor removal at disease onset. Nine patients had 2 or more relapse events (23.1\%; range: 2-3 episodes). Of the 36 patients who underwent lumbar puncture, CSF antibodies were detected in 34 (87.2\%). All 39 patients with relapse underwent serum antibody monitoring, and 13 of 39 (33.3\%) patients showed elevated antibody titers at relapse during serial serum testing. MRI was abnormal in only $8(20.5 \%)$ patients with relapse, and no ovarian teratoma was detected in the patients at relapse.

In relapsing patients with data available at both the first and second events, $30(76.9 \%)$ of 39 relapses were less severe than the initial episodes (figure 2). The mRS score at the second event was significantly lower (median mRS: 2, mean: 2.38 , range: $2-4$ ) than that at onset (median mRS: 4 , mean: 3.87 , range: $2-5$ ). The rate of ICU admission was much lower at the second event (1/39) than at the initial event (7/39), and symptom expression was overall more limited at the second event than at the first event (table e-3, links.lww.com/NXI/ A412). The initial median hospitalization duration was 29 (range: 6-82) days, and the subsequent hospitalization duration was 11 (range: 4-38) days, which was shorter than the initial duration. 
Table 2 Factors Associated With Poor Clinical Outcomes at the 12-Month Follow-up in 225 Patients With Anti-NMDA Receptor Encephalitis

\begin{tabular}{|c|c|c|c|c|}
\hline & \multicolumn{2}{|l|}{ No. of patients (\%) } & \multicolumn{2}{|c|}{ Univariate analysis } \\
\hline & Favorable outcome $(n=191)$ & Poor outcome $(n=34)$ & $Z$ value & $p$ Value \\
\hline Age, y, median (range) & $27(9-78)$ & $24(12-71)$ & 0.06 & 0.79 \\
\hline Sex (female) & $86(44.5)$ & $18(52.9)$ & 0.72 & 0.39 \\
\hline \multicolumn{5}{|l|}{ Symptoms ${ }^{a}$} \\
\hline Fever & $66(34.5)$ & $18(52.9)$ & 0.72 & 0.39 \\
\hline Headache & $71(37.2)$ & $12(35.3)$ & 0.04 & 0.83 \\
\hline Dyskinesias and movement disorders & $73(38.2)$ & $17(50.0)$ & 0.89 & 0.34 \\
\hline Ataxia & $5(2.6)$ & $1(2.9)$ & 0.01 & 0.91 \\
\hline Limb weakness & $2(1.0)$ & $1(2.9)$ & 0.93 & 0.33 \\
\hline Limb numbness & $2(1.5)$ & $1(2.9)$ & 0.72 & 0.39 \\
\hline Cognitive disorder & $128(67.0)$ & $20(58.8)$ & 0.85 & 0.35 \\
\hline Dizziness & $12(6.3)$ & $3(8.8)$ & 0.29 & 0.58 \\
\hline Disturbance of consciousness & $76(39.8)$ & $23(67.6)$ & 8.49 & 0.004 \\
\hline Sleep disorders & $70(36.6)$ & $15(44.1)$ & 0.68 & 0.40 \\
\hline Seizures & 147 (76.9) & $26(76.4)$ & 0.004 & 0.95 \\
\hline Focal onset & $124(64.9)$ & $21(61.7)$ & 0.15 & 0.69 \\
\hline Generalized onset & $71(37.1)$ & 19 (55.9) & 0.04 & 0.84 \\
\hline Epileptic state & $52(27.2)$ & $15(44.1)$ & 3.35 & 0.05 \\
\hline Psychosis & $174(91.1)$ & $30(88.2)$ & 0.27 & 0.59 \\
\hline Mania & $82(42.9)$ & $17(50.0)$ & 0.42 & 0.51 \\
\hline Depression & $24(12.5)$ & $1(2.9)$ & 2.24 & 0.13 \\
\hline Hallucinations & $160(83.7)$ & $27(89.4)$ & 0.38 & 0.53 \\
\hline Autonomic dysfunction, $\geq 1$ of the following & $93(48.7)$ & $19(55.8)$ & 0.24 & 0.62 \\
\hline Hyperhidrosis & $85(44.5)$ & $19(55.9)$ & 1.48 & 0.22 \\
\hline Tachycardia & $49(25.6)$ & $8(23.5)$ & 0.06 & 0.79 \\
\hline Urinary retention or hesitation & $40(20.9)$ & $11(32.3)$ & 2.10 & 0.14 \\
\hline Constipation & $39(20.4)$ & $8(23.5)$ & 0.44 & 0.68 \\
\hline Hyponatremia & $31(16.2)$ & $5(14.7)$ & 0.05 & 0.82 \\
\hline Central hypoventilation & $29(15.2)$ & $10(29.4)$ & 2.32 & 0.04 \\
\hline Admission to the intensive care unit & $27(14.1)$ & $3(8.8)$ & 0.69 & 0.40 \\
\hline \multicolumn{5}{|l|}{ Auxiliary examinations } \\
\hline MRI total with abnormal findings & $78(40.8)$ & $15(44.1)$ & 0.09 & 0.75 \\
\hline \multicolumn{5}{|l|}{ CSF analysis with abnormal findings } \\
\hline Intracranial open pressure $\left(\mathrm{mmH}_{2} \mathrm{O}\right)$ & $58(30.3)$ & $15(44.1)$ & 2.44 & 0.18 \\
\hline WBC $\left(\times 10^{6} / L\right)$ & $91(47.6)$ & $22(64.7)$ & 3.63 & 0.05 \\
\hline Protein $(g / L)$ & $53(27.7)$ & $9(26.4)$ & 0.02 & 0.87 \\
\hline EEG with abnormal findings & $147(76.9)$ & $23(67.6)$ & 0.03 & 0.85 \\
\hline
\end{tabular}


Table 2 Factors Associated With Poor Clinical Outcomes at the 12-Month Follow-up in 225 Patients With Anti-NMDA Receptor Encephalitis (continued)

\begin{tabular}{|c|c|c|c|c|}
\hline & \multicolumn{2}{|l|}{ No. of patients (\%) } & \multicolumn{2}{|c|}{ Univariate analysis } \\
\hline & Favorable outcome $(n=191)$ & Poor outcome $(n=34)$ & $Z$ value & $p$ Value \\
\hline Tumor & $29(15.1)$ & $7(20.5)$ & 0.62 & 0.43 \\
\hline Teratoma & $21(10.9)$ & $4(11.7)$ & 0.10 & 0.89 \\
\hline \multicolumn{5}{|l|}{ Acute immunotherapy } \\
\hline First immunotherapy day $\geq 30 \mathrm{~d}$ from onset & $79(41.3)$ & $14(41.2)$ & 0.01 & 0.98 \\
\hline Treatment modalities & & & 1.64 & 0.53 \\
\hline IVMP alone & $25(13.1)$ & $2(5.9)$ & & \\
\hline IVIg alone & $64(33.5)$ & $14(41.1)$ & & \\
\hline IVMP combined with IVIg & $94(49.2)$ & $18(52.9)$ & & \\
\hline No treatment & $8(4.2)$ & $0(0.0)$ & & \\
\hline$\geq 3$ different immune therapies at the first event & $16(6.6)$ & $3(14.9)$ & 0.93 & 0.42 \\
\hline
\end{tabular}

Abbreviations: IVIg = IV immunoglobulin; IVMP = IV methylprednisolone; $\mathrm{WBC}=$ white blood cell.

Bold entries indicate $p<0.05$.

${ }^{a}$ The cumulative symptoms during the first month of the disease.

At the second disease event (first relapse), all 39 patients with relapse received immunotherapy and underwent reinitiation of the first-line immunotherapy, and among these patients, only 8 received second-line agents (RTX: 4; CTX: 4). There was no significant difference in further relapse risk between the patients treated with and without second-line immunotherapy $(3 / 8[37.5 \%]$ vs $6 / 31$ [19.3\%], Fisher's exact test: $p=$ 0.35 ). Seven patients were also given long-term MMF, and 2 patients were given long-term tacrolimus. There was also no significant difference in the occurrence of subsequent relapse between MMF/tacrolimus-treated patients and other patients $(3 / 9[33.3 \%]$ vs $6 / 30[20 \%]$, Fisher's exact test: $p=0.7)$. However, 1 patient experienced a second relapse during the MMF withdrawal period. The detailed disease and treatment courses for patients with relapse are shown in figure 2 .

Cox regression analysis suggested that the female sex (hazard ratio [HR]: $0.42,95 \%$ CI: $0.20-0.87 ; p=0.02$, figure $3 \mathrm{~A}$ ) and a delay in treatment (HR: $0.41,95 \%$ CI: $0.21-0.78 ; p=0.005$, figure $3 \mathrm{~B}$ ) were significantly associated with an increased risk of relapse. The factors predictive of survival from the first relapse were verified by Cox proportional hazard analysis and are presented as a forest plot in figure e-1 (links.lww.com/ NXI/A410).

To avoid inclusion of potential viral infection or incomplete initial treatment as relapse cases, we separated the patients who relapsed early with antibody negativity, suggesting pseudorelapse or incomplete initial treatment (within 2-6 months; $\mathrm{n}=13$ ) from those who relapsed later with autoantibodies in their CSF (and therefore may be more truly representative of relapse; $n=26$ ). Examining the subsets separately revealed the same predictive factors for all clinical relapse patients and for patients who relapsed late (female [HR: 0.30, 95\% CI: 0.16-0.79; $p=0.01$ ] and experienced delayed treatment [HR: $0.20,95 \% \mathrm{CI}: 0.09-0.44 ; p=$ $0.0001]$ ). However, the female sex and delayed treatment predictive factors were not significantly different for patients who relapsed early. Although neither nontumor (HR: 0.20, 95\% CI: 0.07-0.57; $p=0.08$, figure e-2A, links.lww.com/ $\mathrm{NXI} / \mathrm{A} 411$ ) nor treatment modalities (no treatment [HR: $0.34,95 \%$ CI: $0.55-2.44 ; p=0.29$, figure e-2B] or patients with more than 3 different immunotherapies [HR: 3.01, 95\% CI: $0.74-12.17 ; p=0.12$, figure $\mathrm{e}-2 \mathrm{C}$ ] at the first onset) were not statistically associated with the reported relapse frequency, all figures were slightly higher in the relapse group.

\section{Discussion}

This study updated the clinical features of patients with antiNMDAR encephalitis in Western China in a large cohort and prospectively reported the long-term functional outcomes and relapses. The main findings of the study are as follows: (1) There were differences in demographic data between our cohort and Western countries, with more males and a lower tumor frequency prevailing in our cohort. The male patients were much younger and showed more abnormal ancillary tests and less frequent tumors than females at disease onset. (2) Symptom presentation varied between children and adults, and most patients achieved substantial recovery during the long-term follow-up, which was in line with the studies of Western countries. The overall prognosis continued to improve through 42 months after onset. (3) The patients with disturbance of consciousness at onset remained in a poor neurologic status in the long term. (4) Relapses occurred in 


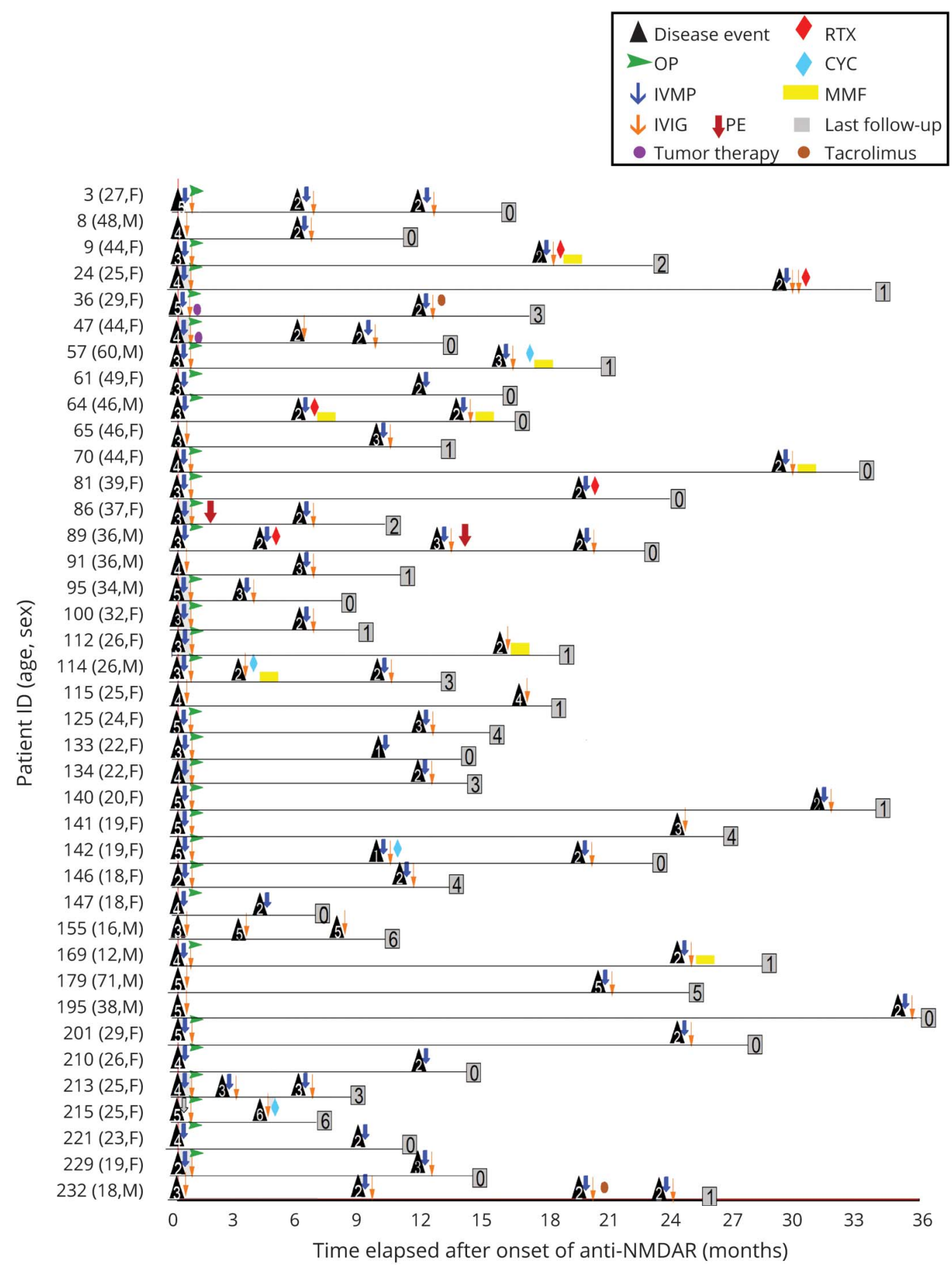

Each line shows 1 disease and the treatment course of the patient with relapse. The black triangles represent a disease event, which included the disease initial onset and relapse episode. The numbers in the triangles and squares represent the modified Rankin Scale scores during the event and at last follow-up, respectively. CYC = cyclophosphamide; IVIG = IV immunoglobulin; IVMP = IV methylprednisolone; MMF = mycophenolate mofetil; NMDAR = NMDA receptor; $\mathrm{OP}=$ oral prednisone; $\mathrm{PE}=$ plasma exchange; $\mathrm{RTX}=$ rituximab.

approximately one-sixth of patients and were generally milder than at onset. In particular, delayed treatment and female sex were risk factors for relapse.

According to most previous studies, patients with antiNMDAR encephalitis are predominantly young females.
However, no sex difference was found in our cohort, which is in agreement with previous Asian countries' reports on adultonset patients in Korea ${ }^{6}$ and on children with anti-NMDAR encephalitis in China. ${ }^{24}$ In addition, we found that male patients presented much younger and less frequently exhibited tumors than females at disease onset. Abnormal MRI, EEG, 
Figure 3 Kaplan-Meier Curves Showing the Frequency of Relapse-free Disease in 244 Patients With AntiNMDA Receptor Encephalitis

A

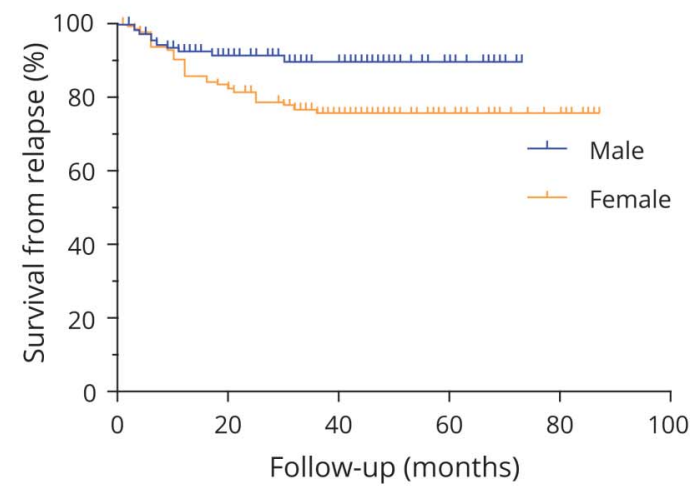

Number at risk

$\begin{array}{rcccc}\text { Male } & 74 & 48 & 19 & 1 \\ \text { Female } & 99 & 58 & 25 & 8 \\ \text { Total } & 173 & 106 & 45 & 9\end{array}$

B

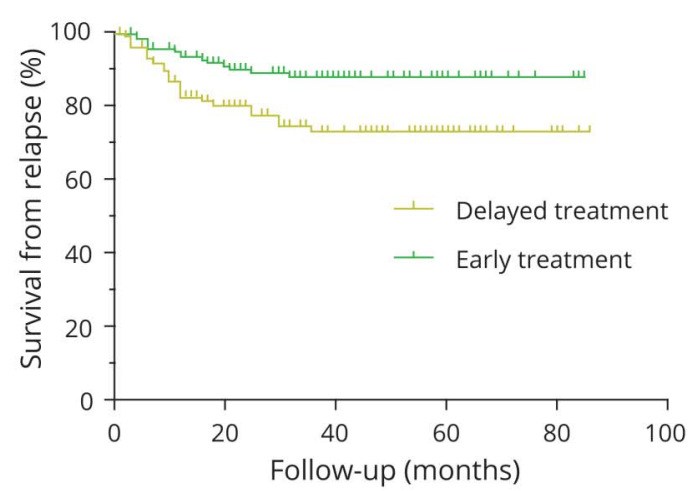

Number at risk

Delayed treatment

Early treatment

Total
66
107

173
44
64

108

$\begin{array}{ll}20 & 5 \\ 22 & 4 \\ 42 & 9\end{array}$

The plots show that patients who $(A)$ were female and $(B)$ had delayed immunotherapy were associated with an increased risk of relapse.

and CSF analyses were reported in $42.21 \%, 77.04 \%$, and $47.54 \%$ of the patients in our study, respectively, and male patients appeared to have more abnormal ancillary test results than female patients. Signals of atypical MRI findings, such as meninx enhancement, pituitary lesions, and nonspecific periventricular white matter lesions, were also included in our study, which did not have clinicopathologic significance (table e-4, links.lww. com/NXI/A412). In our study, many more male patients lived in rural areas, were exposed to poor sanitation and living conditions, and were susceptible to viral infection. In addition, most male patients had smoking habits. All these factors might be associated with frequent ancillary test abnormalities. Compared with that in Western study populations, ${ }^{3,7,25}$ the prevalence of tumors ( $8 \%$ before $2015^{10}$ and $15.57 \%$ through 2019) was still much lower in our study, but it was similar to that reported in other Asian populations (Lim et al., 22.7\%; Xu et al., 19.5\%; and Wang et al., 1.9\%). ${ }^{6,10,14,26}$ These phenomena and discrepancies between different populations thereby indicate that genetic background characteristics (e.g., sex hormones, tumors, and human leukocyte antigens) may attribute to susceptibility to anti-NMDAR encephalitis, and viral infection could also trigger the disease. ${ }^{5,7,26-29}$

Symptom presentation varied between children and adults. The most common clinical profile of the adolescents with anti-NMDAR encephalitis in our study was more neurologic disorders (e.g., fever, sleep disorder, seizures, and disturbance of consciousness), whereas adults presented with more psychiatric and cognitive disorders, which aligns with previous findings. ${ }^{7,24,28}$ In a previous study, ataxia, dyskinesias, and movement disorders were more frequently exhibited in children. However, there were no differences in our study, probably because our cohort did not include patients younger than 9 years. We reported that $48.4 \%$ of patients displayed autonomic symptoms, and other studies on anti-NMDAR encephalitis also reported elevated incidences of autonomic dysfunction (Titulaer: 37\%-48\%; Dalmau: 69\%). ${ }^{5,7}$

In this series of patients, $84.8 \%$ showed clinical improvement within 4 weeks after beginning immunotherapy, and $80.7 \%$ and $85.7 \%$ exhibited substantial recovery (i.e., mild or no residual symptoms) at 12 and 24 months, respectively. The overall prognosis continued to improve through 42 months after onset, which also agrees with the findings in other previous studies (75\%-93\%). ${ }^{7,14}$ In Western countries, general clinical improvements of $50 \%-60 \%$ within 4 weeks have been reported. This is likely because the baseline characteristics of the Western cohort (the median $\mathrm{mRS}$ on admission was 5 , and the ICU admission rate was $50 \%-77 \%$ ) were more severe than those in our cohort (the median baseline mRS was 4 , and only $15 \%$ were admitted to the ICU). Thus, our results might not be applicable to Western populations. Moreover, different populations with varied genetic backgrounds and other external factors (e.g., lifestyle, metabolism, and diet) might also respond differently to treatment. Therefore, more research is required to understand the mechanisms underlying disease initiation, aggravation, and progression in different populations.

The mortality of anti-NMDAR encephalitis in our cohort was also low (only $6.9 \%$ in our study; $7 \%$ in the study by Titulaer ${ }^{7}$ ). In previous studies, the predictors of good functional outcomes were the lack of ICU admission and early treatment, ${ }^{7}$ whereas the poor functional outcome predictors were memory deficits, disturbance of consciousness, young age, and high CSF and serum titers. ${ }^{6,7,13}$ In our study, disturbance of consciousness (an indication of ICU admission) was an independent predictor of poor functional outcomes, which was in line with previous research, whereas young age and cognitive disorders did not predict poor functional outcomes. Our study showed that patients of older ages at disease onset had higher frequencies of ICU admission, and older age was associated with worse functional outcomes. However, only children from 9 years of age and older were included because younger children were all referred to other children's 
specialist hospitals. This fact might also relate to the lack of significance between being of a younger age and poor functional outcome.

Our study showed a relapse rate of $15.9 \%$. Most (82.0\%) patients experienced an initial relapse during the first 24 months. Titulaer et al. followed their cohort for a median time of 2 years, and $12 \%$ of the patients with anti-NMDAR encephalitis experienced clinical relapse. The relapse rate reported in other studies varied between $20 \%$ and $25 \%,{ }^{12,13}$ even reaching $36.4 \% .{ }^{14}$ With regard to treatment modalities, the risk of further relapse was elevated in those who did not receive immunotherapy at onset in the cohort described by Gabilondo et al. ${ }^{30}$ and in patients who did not receive aggressive immunotherapies at the first episode as described by Nosadini et al. ${ }^{21}$; these results also echoed those observed in our cohort (delayed treatment [HR: 0.41, 95\% CI: $0.20-0.75 ; p=0.01]$; figure $3 \mathrm{~B}$ ). Therefore, timely and aggressive immunotherapies might be beneficial for patients with anti-NMDAR encephalitis. Notably, females had an increased risk of recurrence in our follow-up study, which indicated that sex hormones and other genetic background factors may also affect susceptibility to relapses. Compared to Western populations, lack of treatment and lack of second-line immunotherapies during the initial episode were not statistically associated with an increased relapse frequency. These differences were probably due to the small number of patients not receiving immunotherapy $(\mathrm{n}=10)$ or using second-line immunotherapies and plasma exchange at onset $(\mathrm{n}=22)$, as well as due to reduced tumor incidences $(\mathrm{n}=38)$, in our cohort.

However, the current proposed definition of clinical relapse is not based on the presence of antibodies in the CSF but rather on observations and manifestations of clinical symptoms. ${ }^{7,12-14,21}$ Therefore, the reported $15.9 \%$ relapse rate in our study, along with other earlier studies (12\%-35\%), might have been diagnosis bias partially if antibodies in the CSF were lacking because the disease is caused by antibodies. In this cohort, we observed 2 patients with antibody negativity and 3 patients without CSF retesting who experienced early relapse (within 3-6 months). These patients performed a clinical relapse but lacked antibody evidence; thus, pseudorelapse may have occurred because of viral infection, incomplete initial treatment, and so on. Therefore, we divided the subjects into those who relapsed early and those who relapsed later in the hope of revealing more convincing predictive factors and potentially better aligning with relapse data from other studies.

In our study, different treatment modalities at disease onset were not considered to be predictors of time to relapse because such an analysis would have increased the false positive rate. In addition, selective treatment bias occurred in our cohort because of the observational and nonrandomized controlled nature of the study. In our cohort, different treatment modalities were statistically associated with sex (as shown in table e-1, links.lww. com/NXI/A412). The mRS scores at baseline were also significantly different among the IV methylprednisolone (IVMP) alone, IVIg alone, and IVIg + IVMP groups (analysis of variance: $p<0.05$ ).

Despite substantial progress in understanding this disease, many questions remain, and our study has several limitations. First, there was no significant difference in the subsequent recurrence rates between second-line and $\mathrm{MMF} /$ tacrolimustreated patients and other patients in our study, and only a relatively small proportion of patients received second-line or long-term noncorticosteroid regimen therapy. This may be because CTX and RTX are off-label drugs in China and patients fear their cost or adverse effects. Second, it is inevitable that in clinical practice, patients with anti-NMDAR encephalitis and neurologic worsening are more likely to be classified as having autoimmune-mediated relapse rather than viral infection. Thus, to validate relapse and rule out other potential causes, we need to both dynamically observe autoantibodies in the CSF and perform PCR testing to detect herpes simplex virus. Finally, mRS as a primary outcome measure might overshadow findings on relapse and opens the study up to criticisms of the relevance of identifying predictors of poor outcomes in the setting of an incomplete outcome measure. Data have shown that mRS outcomes do not correlate with cognitive, behavioral, psychosocial, or quality of life outcomes. ${ }^{11,17,19}$ To better assess and detect potential risk factors related to prolonged cognitive deficits, neuropsychiatric symptoms, or seizure recurrence symptoms, using a more specific questionnaire and detailed evaluations is necessary (e.g., Neuropsychiatric Inventory, Cognitive Impairment Rating Scale, Montreal Cognitive Assessment, and National Hospital Seizure Severity Scale). Despite these limitations, we describe the clinical profiles, long-term outcomes, and relapse in the largest group of Chinese patients with anti-NMDAR encephalitis in China $(\mathrm{n}=244)$ examined to date, which promotes a deeper understanding of the prognosis of this disease.

\section{Acknowledgment}

The authors thank all participants for their participation in this study.

\section{Study Funding}

This study was supported by the National Science Foundation of China (grants 81671291, 81420108014, and 81971213) and the National Key R\&D Program of China (2018YFC1312300).

\section{Disclosure}

None of the authors have any conflicts of interest to disclose. Go to Neurology.org/NN for full disclosures.

\section{Publication History}

Received by Neurology: Neuroimmunology \& Neuroinflammation July 31, 2020. Accepted in final form December 3, 2020. 
Appendix Authors

\begin{tabular}{|c|c|c|}
\hline Name & Location & Contribution \\
\hline $\begin{array}{l}\text { Xue } \\
\text { Gong, } \\
\text { MD }\end{array}$ & $\begin{array}{l}\text { Department of Neurology, } \\
\text { West China Hospital, Sichuan } \\
\text { University, Chengdu, China }\end{array}$ & $\begin{array}{l}\text { Designed and } \\
\text { conceptualized the study; } \\
\text { analyzed the data; and } \\
\text { drafted the manuscript for } \\
\text { intellectual content }\end{array}$ \\
\hline
\end{tabular}

\begin{tabular}{lll}
\hline $\begin{array}{l}\text { Chu } \\
\text { Chen, } \\
\text { MD }\end{array}$ & $\begin{array}{l}\text { Department of Neurology, } \\
\text { West China Hospital, Sichuan } \\
\text { University, Chengdu, China }\end{array}$ & Collected the data \\
\hline Xu Liu, \\
MD, PhD & $\begin{array}{l}\text { Department of Neurology, } \\
\text { West China Hospital, Sichuan } \\
\text { University, Chengdu, China }\end{array}$ & Collected the data \\
& &
\end{tabular}

Jingfang Department of Neurology, Collected the data

Lin, MD West China Hospital, Sichuan University, Chengdu, China

\begin{tabular}{|c|c|c|}
\hline $\begin{array}{l}\text { Aiqing } \\
\text { Li, MD }\end{array}$ & $\begin{array}{l}\text { Department of Neurology, } \\
\text { West, ChinaChina Hospital, } \\
\text { Sichuan University, } \\
\text { Chengdu, China }\end{array}$ & Collected the data \\
\hline $\begin{array}{l}\text { Kundian } \\
\text { Guo, MD }\end{array}$ & $\begin{array}{l}\text { Department of Neurology, } \\
\text { West China Hospital, Sichuan } \\
\text { University, Chengdu, China }\end{array}$ & Collected the data \\
\hline $\begin{array}{l}\text { Dong } \\
\text { Zhou, } \\
\text { MD, PhD }\end{array}$ & $\begin{array}{l}\text { Department of Neurology, } \\
\text { West China Hospital, Sichuan } \\
\text { University, Chengdu, China }\end{array}$ & $\begin{array}{l}\text { Designed, conceptualized, } \\
\text { and revised the study }\end{array}$ \\
\hline $\begin{array}{l}\text { Zhen } \\
\text { Hong, } \\
\text { MD, PhD }\end{array}$ & $\begin{array}{l}\text { Department of Neurology, } \\
\text { West China Hospital, Sichuan } \\
\text { University, Chengdu, China }\end{array}$ & $\begin{array}{l}\text { Designed, conceptualized, } \\
\text { and revised the study }\end{array}$ \\
\hline
\end{tabular}

\section{References}

1. Dalmau J, Rosenfeld MR. Autoimmune encephalitis update. Neuro Oncol 2014;16: 771-778. doi: 10.1093/neuonc/nou030.

2. Dalmau J, Bataller L. Limbic encephalitis: the new cell membrane antigens and a proposal of clinical-immunological classification with therapeutic implications [in Spanish]. Neurologia 2007;22:526-537.

3. Dalmau J, Gleichman AJ, Hughes EG, et al. Anti-NMDA-receptor encephalitis: case series and analysis of the effects of antibodies. Lancet Neurol 2008;7:1091-1098. doi: 10.1016/S1474-4422(1008)70224-2.

4. Dalmau J, Tüzün E, Wu HY, et al. Paraneoplastic anti-N-methyl-D-aspartate receptor encephalitis associated with ovarian teratoma. Ann Neurol 2007;61:25-36. doi: 10. 1002/ana.21050.

5. Dalmau J, Lancaster E, Martinez-Hernandez E, Rosenfeld MR, Balice-Gordon R. Clinical experience and laboratory investigations in patients with anti-NMDAR encephalitis. Lancet Neurol 2011;10:63-74. doi: 10.1016/S1474-4422(1010)70253-2.

6. Lim JA, Lee ST, Jung KH, et al. Anti-N-methyl-d-aspartate receptor encephalitis in Korea: clinical features, treatment, and outcome. J Clin Neurol 2014;10:157-161. doi: 10.3988/jcn.2014.10.2.157.

7. Titulaer MJ, McCracken L, Gabilondo I, et al. Treatment and prognostic factors for long-term outcome in patients with anti-NMDA receptor encephalitis: an observational cohort study. Lancet Neurol 2013;12:157-165. doi: 110.1016/S14744422(1012)70310-1.

8. Zekeridou A, Karantoni E, Viaccoz A, et al. Treatment and outcome of children and adolescents with N-methyl-D-aspartate receptor encephalitis. J Neurol 2015;262: 1859-1866. doi: 10.1007/s00415-015-7781-9.
9. Irani SR, Bera K, Waters $\mathrm{P}$, et al. N-methyl-D-aspartate antibody encephalitis: temporal progression of clinical and paraclinical observations in a predominantly nonparaneoplastic disorder of both sexes. Brain 2010;133:1655-1667. doi: 10.1093/ brain/awq113.

10. Wang W, Li JM, Hu FY, et al. Anti-NMDA receptor encephalitis: clinical characteristics, predictors of outcome and the knowledge gap in southwest China. Eur J Neurol 2016;23:621-629. doi: 10.1111/ene.12911.

11. Liu X, Zhang L, Chen C, et al. Long-term cognitive and neuropsychiatric outcomes in patients with anti-NMDAR encephalitis. Acta Neurol Scand 2019;140:414-421. doi: 10.1111/ane.13160.

12. Gabilondo I, Saiz A, Galán L, et al. Analysis of relapses in anti-NMDAR encephalitis. Neurology 2011;77:996-999. doi: 10.1212/WNL.0b013e31822cfc6b.

13. Gresa-Arribas N, Titulaer MJ, Torrents A, et al. Antibody titres at diagnosis and during follow-up of anti-NMDA receptor encephalitis: a retrospective study. Lancet Neurol 2014;13:167-177. doi: 10.1016/S1474-4422(13)70282-5.

14. Xu X, Lu Q, Huang Y, et al. Anti-NMDAR encephalitis: a single-center, longitudinal study in China. Neurol Neuroimmunol Neuroinflamm 2020;7:e633. doi: 10.1212/ NXI.0000000000000633. Print 0000000000002020 Jan.

15. Zhang L, Lu Y, Xu L, et al. Anti-N-methyl-D-aspartate receptor encephalitis with accompanying ovarian teratoma in female patients from East China: clinical features, treatment, and prognostic outcomes. Seizure 2020;75:55-62. doi: 10.1016/j.seizure. 2019.12.016.

16. Wang R, Lai XH, Liu X, et al. Brain magnetic resonance-imaging findings of anti-Nmethyl-D-aspartate receptor encephalitis: a cohort follow-up study in Chinese patients. J Neurol 2018;265:362-369. doi: 10.1007/s00415-017-8707-5.

17. Liu X, Yan B, Wang R, et al. Seizure outcomes in patients with anti-NMDAR encephalitis: a follow-up study. Epilepsia 2017;58:2104-2111. doi: 10.1111/epi. 13929.

18. Graus F, Titulaer MJ, Balu R, et al. A clinical approach to diagnosis of autoimmune encephalitis. Lancet Neurol 2016;15:391-404. doi: 10.1016/S1474-4422(15) 00401-9.

19. van Swieten JC, Koudstaal PJ, Visser MC, Schouten HJ, van Gijn J. Interobserver agreement for the assessment of handicap in stroke patients. Stroke 1988;19:604-607. doi: 10.1161/01.str.19.5.604.

20. Armangue T, Titulaer MJ, Málaga I, et al. Pediatric anti-N-methyl-D-aspartate receptor encephalitis-clinical analysis and novel findings in a series of 20 patients. J Pediatr 2013;162:850-856.e852. doi: 10.1016/j.jpeds.2012.10.011.

21. Nosadini M, Granata T, Matricardi S, et al. Relapse risk factors in anti-N-methyl-Daspartate receptor encephalitis. Dev Med Child Neurol 2019;61:1101-1107. doi: 10. $1111 /$ dmcn. 14267

22. Schemper M, Smith TL. A note on quantifying follow-up in studies of failure time. Control Clin Trials 1996;17:343-346. doi: 10.1016/0197-2456(96)00075-x.

23. Peduzzi P, Concato J, Kemper E, Holford TR, Feinstein AR. A simulation study of the number of events per variable in logistic regression analysis. J Clin Epidemiol 1996;49: 1373-1379. doi: 10.1016/s0895-4356(96)00236-3.

24. Wang Y, Zhang W, Yin J, et al. Anti-N-methyl-d-aspartate receptor encephalitis in children of Central South China: clinical features, treatment, influencing factors, and outcomes. J Neuroimmunol 2017;312:59-65. doi: 10.1016/j. jneuroim.2017.09.005.

25. Florance NR, Davis RL, Lam C, et al. Anti-N-methyl-D-aspartate receptor (NMDAR) encephalitis in children and adolescents. Ann Neurol 2009;66:11-18. doi: 10.1002/ ana.21756.

26. Huang X, Fan $\mathrm{C}, \mathrm{Wu}$ J, et al. Clinical analysis on anti-N-methyl-D-aspartate receptor encephalitis cases: Chinese experience. Int J Clin Exp Med 2015;8:18927-18935. eCollection 12015

27. Armangue T, Spatola M, Vlagea A, et al. Frequency, symptoms, risk factors, and outcomes of autoimmune encephalitis after herpes simplex encephalitis: a prospective observational study and retrospective analysis. Lancet Neurol 2018;17:760-772. doi 10.1016/S1474-4422(18)30244-8.

28. Dalmau J, Armangué T, Planagumà $\mathrm{J}$, et al. An update on anti-NMDA receptor encephalitis for neurologists and psychiatrists: mechanisms and models. Lancet Neurol 2019;18:1045-1057. doi: 10.1016/S1474-4422(19)30244-3.

29. Fan $\mathrm{S}, \mathrm{Xu} \mathrm{Y}$, Ren $\mathrm{H}$, et al. Comparison of myelin oligodendrocyte glycoprotein (MOG)-antibody disease and AQP4-IgG-positive neuromyelitis optica spectrum disorder (NMOSD) when they co-exist with anti-NMDA (N-methyl-D-aspartate) receptor encephalitis. Mult Scler Relat Disord 2018;20:144-152. doi: 10.1016/j. msard.2018.01.007.

30. Titulaer MJ, McCracken L, Gabilondo I, et al. Late-onset anti-NMDA receptor encephalitis. Neurology 2013;81:1058-1063. doi: 10.1212/WNL.0b013e3182a4a49c. 


\title{
Neurology \\ Neuroimmunology \& Neuroinflammation
}

\author{
Long-term Functional Outcomes and Relapse of Anti-NMDA Receptor Encephalitis: A \\ Cohort Study in Western China \\ Xue Gong, Chu Chen, Xu Liu, et al. \\ Neurol Neuroimmunol Neuroinflamm 2021;8; \\ DOI 10.1212/NXI.0000000000000958
}

This information is current as of February 15, 2021

\begin{abstract}
Updated Information \&
Services

References

Subspecialty Collections

Permissions \& Licensing

Reprints

including high resolution figures, can be found at:

http://nn.neurology.org/content/8/2/e958.full.html

This article cites 30 articles, 1 of which you can access for free at: http://nn.neurology.org/content/8/2/e958.full.html\#\#ref-list-1

This article, along with others on similar topics, appears in the following collection(s):

Clinical trials Observational study (Cohort, Case control) dy_cohort_case_control its entirety can be found online at:

http://nn.neurology.org/misc/about.xhtml\#permissions

Information about ordering reprints can be found online:

http://nn.neurology.org/misc/addir.xhtml\#reprintsus
\end{abstract}

http://nn.neurology.org//cgi/collection/clinical_trials_observational_stu

Information about reproducing this article in parts (figures,tables) or in

Neurol Neuroimmunol Neuroinflamm is an official journal of the American Academy of Neurology.

Published since April 2014, it is an open-access, online-only, continuous publication journal. Copyright

Copyright $\odot 2021$ The Author(s). Published by Wolters Kluwer Health, Inc. on behalf of the American

Academy of Neurology.. All rights reserved. Online ISSN: 2332-7812.

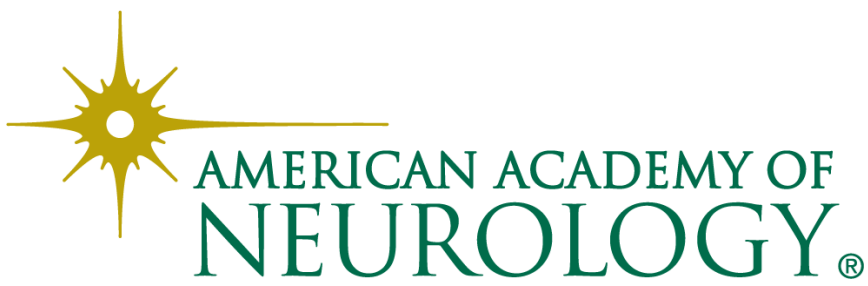

